\title{
CORRECTION
}

\section{Correction to: Friction and Plasticity in Contacts Between Amorphous Solids}

\author{
Binquan Luan ${ }^{1}$ (D) Mark O. Robbins ${ }^{2}$
}

(c) Springer Science+Business Media, LLC, part of Springer Nature 2021, corrected publication 2021

\section{Correction to: Tribology Letters (2021) 69:51 https://doi.org/10.1007/s11249-021-01429-7}

The original version of this article unfortunately contained a mistake in the graphical abstract.

The correct version is given below.

The original article has been corrected.

The original article can be found online at https://doi.org/10.1007/ s11249-021-01429-7.

\section{Binquan Luan}

bluan@us.ibm.com

1 Computational Biological Center, IBM Thomas J. Watson Research, Yorktown Heights, NY 10598, USA

2 Department of Physics \& Astronomy, Johns Hopkins University, Baltimore, MD 21218, USA 
Graphical Abstract The origins of friction and energy dissipation at an atomic scale are not well understood. Prof. Robbins designed large-scale, molecular-dynamics simulations to investigate interfacial friction between amorphous solids. Importantly, the friction force was found to be proportional to the number of plastic-deformation events, i.e. the energy is dissipated when overcoming an energy barrier between two plastically deformed atomic structures. Some original sketches can be seen here, taken directly from his notes, showing his visions for the project

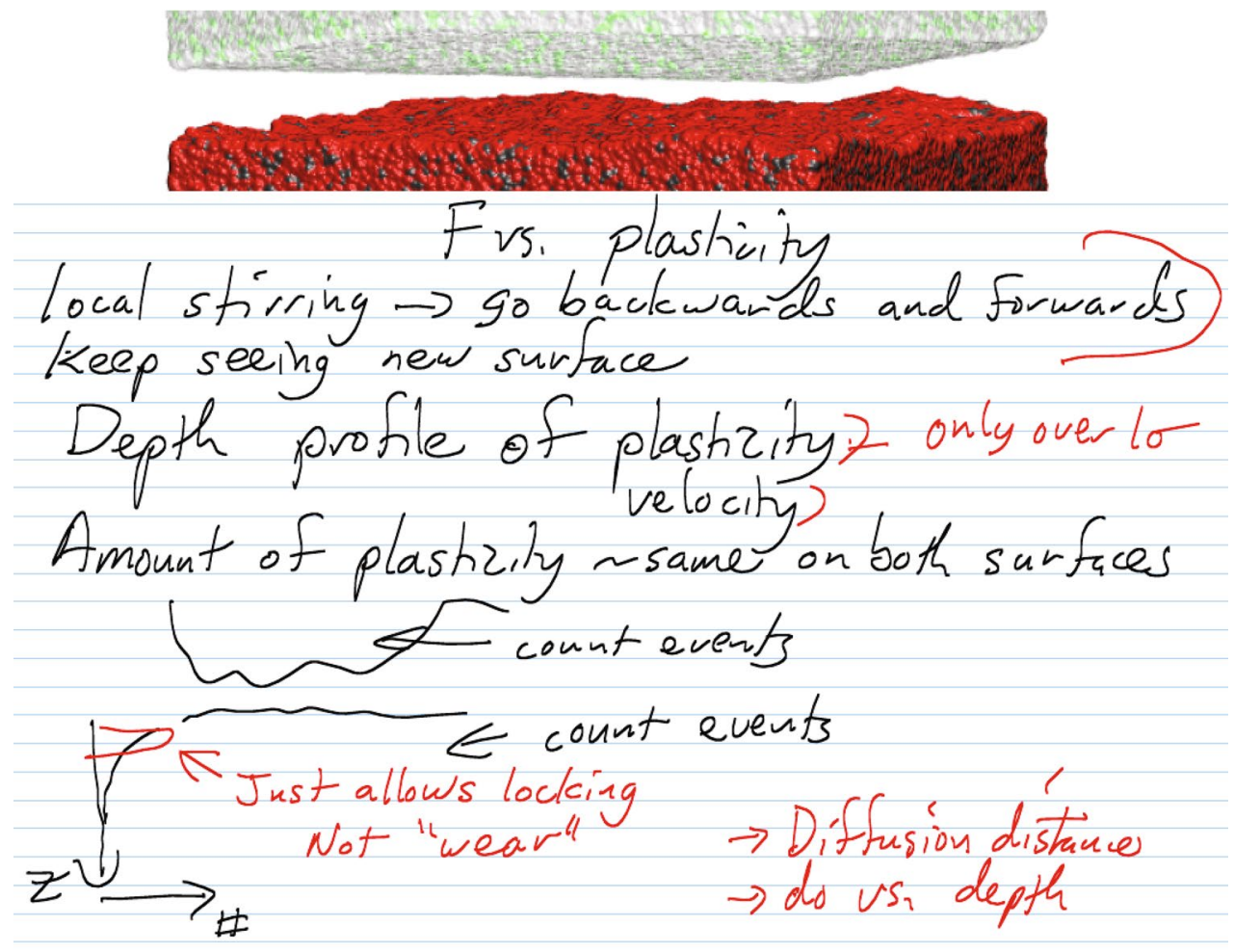

Publisher's Note Springer Nature remains neutral with regard to jurisdictional claims in published maps and institutional affiliations. 